\title{
Verification of a Multiprocessor Cache Protocol using Simulation Relations and Higher-Order Logic (Summary)
}

\author{
Paul Loewenstein* (Mitsubishi Electronics America) and \\ David L. Dill ${ }^{\dagger}$ (Stanford University)
}

\begin{abstract}
We present a formal verification method for concurrent systems. The technique is to show a correspondence between state machines representing an implementation and specification behaviour. The correspondence is called a simulation relation, and is particularly well-suited for theorem-provers. Since the method does not rely on enumerating all the states, it can be applied to systems with an infinite or unknown number of states. This substantially expands the class of hardware designs that can be formally verified. The method is illustrated by proving the correctness of a particularly subtle example which is likely to be of increasing importance: a directory-based multiprocessor cache protocol. The proof is carried out using the HOL ("higher-order logic") theorem-prover.
\end{abstract}

\section{Introduction}

It has been shown that formal verification of certain types of non-trivial hardware designs using theorem-provers is possible. Indeed, several complete (albeit simple) processors have been formally verified $[4,3,10,17]$ using theorem-proving. But before theorem-proving techniques can be applied to most hardware designs, the problem of modelling and verifying concurrent behaviour must be solved. Unforseen interactions among concurrent systems are one of the major source of bugs in hardware designs today. Significantly, architectural features that depend on concurrent interaction have been omitted from almost all formally verified processor designs. Such features include caches, pipelining, interrupts, and page mapping. Concurrency is crucial for high performance, so its use will continue to increase. In particular, multiprocessor architectures are certain to be a major source of complicated concurrent hardware designs. This paper describes a successful attempt to verify a high-level concurrent hardware design.

*Work performed at Stanford University while visiting scientist for National Semiconductor Corporation.

Supported by NSF grant number MIP-8858807 and DARPA contract number N00014-87-K-0828. 
The cache coherence protocol of our example is directory-based. The main idea behind directory-based cache coherence protocols is to maintain a partial model of cache contents in a central directory (or directories). When, for example, an address is written for the first time in one cache, the directory can send "invalidate" messages to the specific caches which have data present at the same address. The advantage is that cache invalidations do not have to be broadcast, eliminating a major bottleneck in large multi-processors.

The protocol involves the interaction of several concurrent agents, namely the individual cache controllers and the directory. In general, models of concurrent systems are fundamentally different from, say models of functional programs. It is not sufficient to model a system as an input/output mapping on data-values or memory states. A model must also capture the sequence of states during a computation, because the interactions with other systems depends crucially on the intermediate states of the computation.

We use a well-known method for verifying concurrent systems based on simulation relations. The implementation (system behaviour) and specification (desired behaviour) are both represented as state graphs. A simulation relation establishes a correspondence between implementation states and specification states. For the example of the paper, we use successive refinement. We transform a higher-level description into an implementation in several steps. A simulation relation is used to show that the automaton after the step implements the automaton before the step.

Previously, there have been proposals for logics and methods for reasoning about concurrent systems too numerous to mention. However, one promising class of methods is to establish a correspondence between two automata (the correspondence can be a single- or multi-valued function, or a relation) $[13,1,12,19,11,16]$. Although some impressive examples have been done by hand these methods have generally not been combined with automatic theorem-proving (the one other example of which we are aware is due to. Nipkow [18]).

The most successful methods for verifying concurrent hardware are state enumeration methods. In essence, these methods automatically find all of the reachable states in a state graph representing the behaviour of a circuit For example, in the model-checking approach, the state graph is compared with temporal logic formulas, which make assertions about the execution paths through the system $[2,7]$. Other techniques compare formal languages of finite automata $[5,6]$.

Of course, the major limitation of state enumeration methods is that they potentially need to inspect all of the states of the system. They all suffer from the state explosion problem - the number of states grows exponentially with the size of the system being verified. They are also generally not good for systems that are partially described. For example, in our description of a cache system, we never specify the number of processes, size of memory, size of data values, or many other parameters of the system. Hence, we have verified the correctness of all the systems that can be obtained by giving values to these parameters. A state enumeration approach would require not only that values be assigned to these parameters, but that the values be very small integers. In some case, one can show that a parameterized design is correct for all parameter values by verifying a few designs with particular values for the parameters. However, these techniques are either highly restricted or it requires human insight to extend the proof from special cases to the general case.

Theorem proving and state enumeration are complementary methods - each is appro- 
priate for a particular class of problems. Eventually, we hope that a combination of both approaches will make hardware design verification economically feasible (and routine).

We carry out proofs using the HOL theorem-prover ("Higher-Order Logic") $[9,8]$. $\mathrm{HOL}$ uses a very expressive logic, in which variables can range over functions as well as values (unlike first-order logic). Our approach is to express everything in HOL: indeed, the implementation and specification state graphs are given as logical formulas. There are several advantages to using HOL. The generality of HOL allowed us to prove theorems not only about hardware, but about mathematics, including the soundness of the method itself. Also, the HOL type system allows proofs about objects without knowing their types. This results in generic results that can easily be reused.

\section{Notation}

For concreteness, we have included some of the theorems and formulas used to describe state graphs. For brevity, we have omitted most of them (for a computer file containing everything, including the proofs, please contact one of the authors). We have modified the notation somewhat to improve readability for readers who are not very familiar with HOL (for a detailed description of the HOL logic, see [8]).

HOL logic reads very much like ordinary predicate logic with a few differences. Variables and constants can range over functions or values, depending on their type. Types are left implicit in this paper; they are explained in the text unless they are obvious from context. Function application is indicated by juxtaposition: $f x$ denotes function $f$ applied to $x$. Often multiple argument functions are curried; $g x y$ denotes a function $g$ applied to $x$, the result (again a function) is then applied to $y$. Informally one can consider $g$ to be merely a multiple argument function. The $(x, y)$ notation is used for pairing (cartesian product). When pairs are used as function arguments, as in $f(x, y)$, the notation appears conventional. Sometimes, (as in this paper), currying and pairing are mixed, as in $g(u, v)(x, y)$. Logical implication is indicated by the $\supset$ operator.

Proven theorems are indicated by the symbol $\vdash$. These have been derived using the mechanical theorem prover, HOL[9], and should be correct apart from errors in typographic transcription. Defined constants are printed in sans serif typeface.

Let $x=y$ in $z[x]$ is syntactic sugar for a term semantically identical to ( $\lambda x . z[x]) y$, which reduces to $z[y]$. It saves repeating a long term $y$ which may have multiple occurrences in $z[y]$.

We have adopted some special conventions for describing sequences and automata. Variables denoting sequences, or functions of time, are emboldened $(e)$, as opposed to other variables $(e)$. When referring to next-state relations, unprimed variables $(s)$ refer to the current state, and primed variables $\left(s^{\prime}\right)$ to the next state. The initial state predicate of an automaton Name, is always Name_P. The next-state relation is Name_N and any invariant is Name.l.

This paper gives an overview of the proof. More details are available in a longer version which appears in [15]. In the remainder of the paper, the next section describes the state-graph model of behaviour and the definition of simulation relations. The third section outlines our proof strategy, which transforms a high-level description of the system through several intermediate representations to the final implementation. The final section discusses the appropriate use of the verification method and future work. 


\section{The Automaton Model}

We regard a digital system as a concurrent process with externally visible variables (corresponding to wires, or bundles of wires) and invisible internal state. A trace is an infinite externally-visible execution history of the system. The behaviour of a system is the set of all possible traces that it can exhibit. This set can be related to a state graph, consisting of of a set of states, a subset of those representing possible initial states, and a next-state relation.

Each state is denoted by a pair $(e, s)$, where $e$ is the visible (or external) part of the state and $s$ is the hidden, or internal, part. A state graph is defined by a pair of predicates, $(P, N): P$ is a unary predicate that defines the the initial states, and $N$ is a binary next-state relation: $N(e, s)\left(e^{\prime}, s^{\prime}\right)$ holds when $\left(e^{\prime}, s^{\prime}\right)$ is a possible successor state to $(e, s)$.

A binary predicate (Labelled State Automaton) $\operatorname{LSA}(P, N) \boldsymbol{e}$ is defined in HOL. It states that $e$ is a trace of the automaton defined by $P$ (initial states) and $N$ (next state relation). In more detail, $e$ is a trace of the automaton if there exists a sequence of hidden states, $s$, such that the first state satisfies $P$, and $N$ holds between every state and the next state in the sequence:

$$
\operatorname{LSA}(P, N) e=(\exists s . P(e 0, s 0) \wedge(\forall t . N(e t, s t)(e(t+1), s(t+1))))
$$

\subsection{Relating two automata}

One automaton implements another if every trace of the first is an trace of the second. A powerful method for showing that this relation holds is to find a simulation nelation between them. A simulation relation is a three-way relation $\operatorname{Res}_{1} s_{2}$, where $\left(e, s_{1}\right)$ is a state of the implementation graph and $\left(e, s_{2}\right)$ is a state of the specification graph (the visible components must be the same in both states). It must satisfy two properties: first, every initial state of the implementation is related by $R$ to some initial state of the specification; and, second, whenever $R e s_{1} s_{2}$ and $N_{1}\left(e, s_{1}\right)\left(e^{\prime}, s_{1}^{\prime}\right)$ both hold, there exists some specification state $\left(e^{\prime}, s_{2}^{\prime}\right)$ such that $N_{2}\left(e, s_{2}\right)\left(e^{\prime}, s_{2}^{\prime}\right)$ and $R e^{\prime} s_{1}^{\prime} s_{2}^{\prime}$ both hold.

To show that one automaton is an implementation of another, it is sufficient to produce a simulation relation from the first to the second. This fact is proven as a theorem in HOL $^{1}$

$$
\begin{aligned}
& \vdash \forall P_{1} N_{1} P_{2} N_{2} . \\
& (\exists R . \\
& \left(\forall e s_{1} \cdot P_{1}\left(e, s_{1}\right) \supset\left(\exists s_{2} . P_{2}\left(e, s_{2}\right) \wedge \text { Re } s_{1} s_{2}\right)\right) \wedge \\
& \quad\left(\forall e e^{\prime} s_{1} s_{1}^{\prime} s_{2} \cdot R e s_{1} s_{2} \wedge N_{1}\left(e, s_{1}\right)\left(e^{\prime}, s_{1}^{\prime}\right) \supset\right. \\
& \left.\left.\left(\exists s_{2}^{\prime} . R e^{\prime} s_{1}^{\prime} s_{2}^{\prime} \wedge N_{2}\left(e, s_{2}\right)\left(e^{\prime}, s_{2}^{\prime}\right)\right)\right)\right) \supset \\
& \left(\forall e . \operatorname{LSA}\left(P_{1}, N_{1}\right) e \supset \operatorname{LSA}\left(P_{2}, N_{2}\right) e\right)
\end{aligned}
$$

The types of $e$ and $s_{1}$ and $s_{2}$ are left open; the theorem therefore holds for finite, countable and uncountable-state automata.

This is a soundness result for our verification method: to prove that one automaton implements another, it is sufficient to supply a simulation relation. The converse would

\footnotetext{
${ }^{1}$ This theorem was derived from the corresponding theorem for a labelled transition automaton presented with proof in [14].
} 


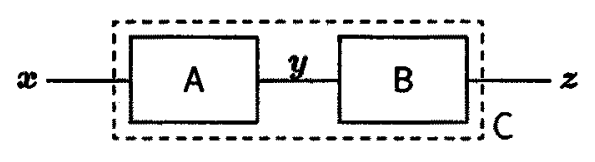

Figure 1: Composition of automata

be that whenever one graph is an implementation of another, there exists a simulation relation. This property (completeness) does not hold. Nevertheless, simulation relations have been adequate for our purposes. Abadi and Lamport have shown that a similar method can be made complete if additional variables are added to the implementation to record the past and predict the future (history ${ }^{2}$ and prophecy variables), a result that is equally applicable to our method, should the addition of variables prove necessary [1].

An invariant is a predicate on individual states. An invariant is satisfied by a state graph if every state that is reachable from an initial state satisfies the invariant. An important method for simplifying proofs is to prove strong invariants before proving more complicated properties. Once an invariant has been proven on a state graph, the invariant can be incorporated into the next-state relation of original state graph without changing the behaviour of the system (this just makes explicit a property that is already implicitly true). This transformation often substantially reduces the amount of case analysis that must be used in proofs of additional properties of the state graph. The correctness of this transformation (although obvious) has been proven in HOL by specialisation of theorem 2 .

\subsection{Composition and Hiding}

Figure 1 shows a parallel composition of two circuits, $A$ and B. Given definitions of the automata for $A$ and $B$, we can write the initial state predicate of $C$ as

$$
\mathrm{C}_{-} \mathrm{P}\left((x, z), s_{1}, s_{2}, y\right)=\mathrm{A} \_\mathrm{P}\left((x, y), s_{1}\right) \wedge \mathrm{B} \_\mathrm{P}\left((y, z), s_{2}\right),
$$

where A_P and B_P are the initial state predicates of the individual machines. Note that $y$ has moved from being a component of the label of the individual machine state, to being a component of the hidden state of the composed machine (composition without hiding can be defined be putting $y$ with $x$ and $z$, if desired). The next-state of $C$ relation is defined similarly.

$$
\begin{aligned}
& \mathrm{C} \_\mathrm{N}\left((x, z), s_{1}, s_{2}, y\right)\left(\left(x^{\prime}, z^{\prime}\right), s_{1}^{\prime}, s_{2}^{\prime}, y^{\prime}\right)= \\
& \quad \mathrm{A} N\left((x, y), s_{1}\right)\left(\left(x^{\prime}, y^{\prime}\right), s_{1}^{\prime}\right) \wedge \mathrm{B} \_\mathrm{N}\left((y, z), s_{2}\right)\left(\left(y^{\prime}, z^{\prime}\right), s_{2}^{\prime}\right)
\end{aligned}
$$

Although it is possible to prove a general schema as a theorem, we found it simpler to merely prove each composition individually by expanding the LSA definition.

\section{Highlights of the Specification and Proof}

This section gives a very high-level overview of the proof. A preliminary task was to define a common master-slave communication channel MS, which is used at every interface where

\footnotetext{
"Our simulation relation approach does not need history variables.
} 
data is read or written. In the figures, communication delay is represented by circled arrows, with the arrow pointing from master to slave.

The actual proof refines an specification of memory behavior, which we call a multiport memory to a memory system consisting of a cache for each processor, a directory, and a main memory (which is also a multi-port memory). The first step is to refine the multi-port memory into what we call a cached multi-port memory. The cached multi-port memory is not directly implementable as specified - it exists only to simplify the proof. The second part of the proof is to refine a component of the cached multi-port memory into a composition of two smaller components. Finally, the directory-based system is formed by merging several of the components into a single directory. This "step" is just an application of composition and hiding. We now discuss these steps in more detail.

\subsection{Multi-port Memory}

We first define the behaviour of an inulti-port memory with no cache, MP_Mem. MP_Mem serves two purposes: MP_Mem is a part of the implementation because a cached memory is just a multi-port memory with a cache in front; MP_Mem is also the specification of the desired behaviour of the cached memory (a correctly functioning cached memory behaves just like an uncached memory).

The per-port components of the multi-port memory are modelled as functions from a port address, $q$, to a value. The type of $q$ is left open so that the proofs are valid for any number of ports. This is possible because none of the consistency proof uses induction; the only induction is over time in the derivation of theorem 2 .

\subsection{Refining the Multi-Port Memory to a Cached Multi-port memory}

We first refine the (uncached) multi-port memory to a simple cached multi-port memory. This involves defining the cached memory and proving that it implements the multi-port memory.

The cached multi-port memory consists of three types of components: a multi-port memory for primary storage, a cache Port_Cache (there is one for each port) and unit EXA which goes between the memory and port caches, whose primary purpose is to enforce mutually-exclusive access by the port caches to the multi-port memory.

Port_Cache looks very much like a single processor cache, except that it has a "window", $s_{d}$, on part of its hidden state. This window is used by EXA both to obtain information about the internal state of the Port_Caches, and to constrain the behaviour of the Port_Caches. It is very important to note that we have provided no explicit mechanism for this two-way transfer of information between Port_Cache and EXA (it is therefore not indicated on figure 2 as a circled arrow). This is an example of a generally useful trick: partitioning the logical description of a design in ways that are not physically implementable. In this case, it makes the proof much easier, and no harm is done because the next step will refine the unimplementable description into an implementable one by repartitioning the system. EXA uses $s_{d}$ to decide when it can grant a cache exclusive access, and also to block access directly. 


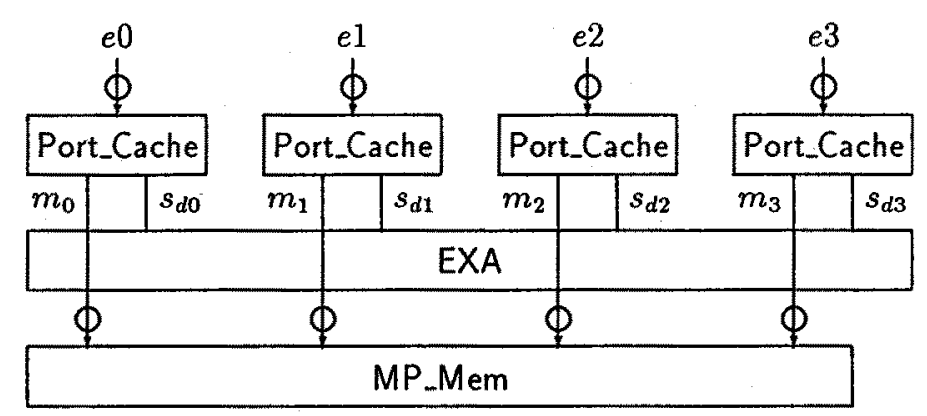

Figure 2: Modified caches with exclusive access control for a multi-port memory

It is then proved, by the use of a simulation relation, that the cached multi-port memory implements the uncached multi-port memory.

\subsection{Splitting the port cache}

The second part of the proof refines the Port_Cache into a composition of two halves: Cache, which is the final (per-port) cache specification, and Port_Dir, which will eventually end up being part of the directory. The two halves communicate via two MS communication channels, one of which carries directory-to-cache requests, and the other carries cache-to-directory requests. It is then proved, by using a simulation relation, that Port_Dir coupled to Cache implements Port_Cache. Despite the fact that this can be carried out on one port in isolation, it is by far the largest part of the total multiport correctness proof. The final system is shown in figure 3.

The directory consists of the EXA and the collection of Port_Dir, which is regarded as a single unit. The division between EXA and Port_Dir in the intermediate representation was only a convenient fiction which may have nothing to do with the actual implementation of the directory. The $s_{d}$ state is now completely enclosed in the directory, so the communication between the different components is entirely via master-slave channels. So, if the individual components are implementable (as we believe they are), the entire system is also implementable.

At this point, we cease refining the specification. If we wanted to carry it further, MP_Mem could be implemented with a memory request arbiter and a single port memory, in which case the arbiter would also become part of the directory. One could also use another level of caching at each processor.

\section{Conclusion}

Before this project we thought that one could perform verification by proceeding top-down from a single specification, deciding on specifications of components and proving that the assembly of components conforms to the specification. Then repeat the process on the components, thus following a "verification tree" all the way to the primitive components. 


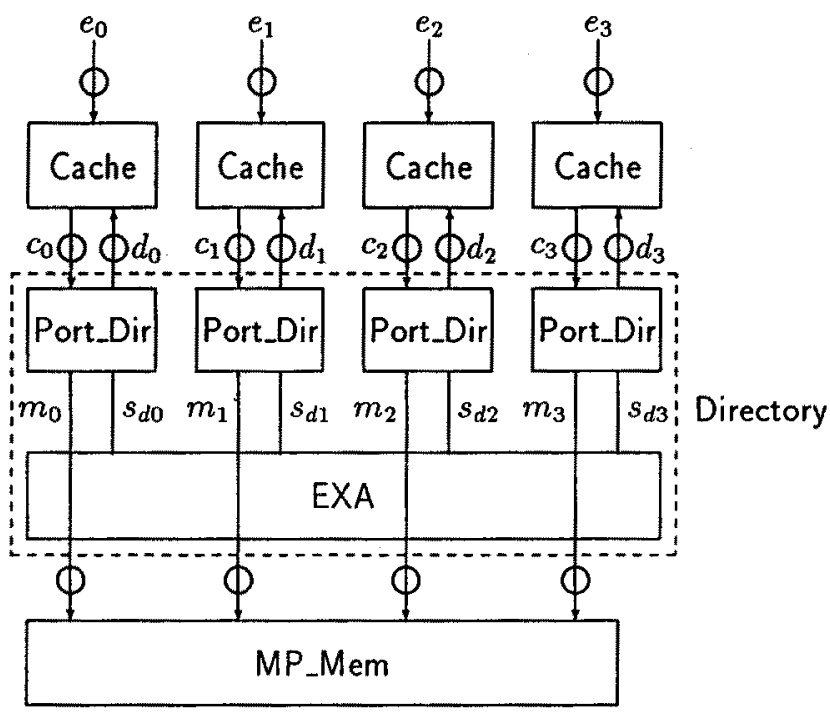

Figure 3: Configuration after splitting the caches

Things are not that simple. For example, modelling the directory state as a memory per cache requires merging, not division, as the proof proceeds down the abstraction hierarchy. The design hierarchy becomes a much more dynamic concept, different compositions being used to derive different properties.

The principle inspiration in the proofs is the finding of the simulation relation, and choosing invariants that are sufficiently strong. The rest of the proof is tedious and boring. To make this a routine way to approach engineering design we need a much improved user interface, much better interactive response time, and better automatic proof procedures than HOL currently offers. The re-running (without interaction) of the proofs in this paper takes about 20 hours on an unloaded Sun $3 / 60$.

The automaton theory used in this paper was derived in a general-purpose theorem prover. The theory required to reason about other aspects of the design (such as Temporal logic for deriving liveness properties), can also be derived in the same environment, thus allowing more complete proof inside a single environment.

By using a suitable powerful logic can prove the correctness of systems with an arbitrarily large state-space, provided that the system is structured to allow a tractable case analysis. 


\section{References}

[1] Martín Abadi and Leslie Lamport. The existence of refinement mappings. SRC Report 29, Digital Equipment Corporation, 1988.

[2] Michael C. Browne, Edmund M. Clarke, David L. Dill, and Bud Mishra. Automatic verification of sequential circuits using temporal logic. IEEE Transactions on Computers, C-35(12):1035-1044, December 1986.

[3] Avra Cohn. Correctness properties of the Viper block model: The second level. In G. Birtwistle and P. A. Subrahmanyam, editors, Current Trends in Hardware Verification and Automated Theorem Proving, pages 1-91. Springer-Verlag, 1989.

[4] Avra. J. Cohn. A proof of correctness of the Viper microprocessor: The first level. In G. Birtwistle and P. A. Subrahmanyam, editors, VLSI Specification, Verification and Synthesis. Kluwer Academic Publishers, 1988.

[5] Srinivas Devadas, Hi Keung Ma, and A. Richard Newton. On the verification of sequential machines at differing levels of abstraction. Memorandum UCB/ERI M86/93, University of California, Berkeley, 1986.

[6] David L. Dill. Trace Theory for Automatic Hierarchical Verification of Speedindependent Circuits. MIT Press, 1989.

[7] Masahirio Fujita, Hedehiko Tanaka, and Tohru Moto-oka. Verification with prolog and temporal logic. In T. Uehara and M. Barbacci, editors, IFIP Sixth Computer Hardware Description Lanquages and their applications, pages 103-114. North Holland Publishing Company, 1983.

[8] Mike Gordon. HOL: A machine oriented formulation of higher-order logic. Technical Report 68, University of Cambridge Computer Laboratory, 1985.

[9] Mike Gordon. HOL: A proof generating system for higher-order logic. In G. Birtwistle and P. A. Subrahmanyam, editors, VLSI Specification, Verification and Synthesis. Kluwer Academic Publishers, 1988.

[10] W. A. Hunt, Jr. The mechanical verification of a microprocessor design. In D. Borrione, editor, From HDL Descriptions to Guaranteed Correct Circuit Designs. North Holland, 1987.

[11] Nils Klarlund and Fred B. Schneider. Verifying safety properties using nondeterministic infinite-state automata. Technical Report TR 89-1037, Cornell University Computer Science Department, 1989.

[12] R. P. Kurshan. Analysis of discrete event coordination. In J.W. de Bakker, W.* P. De Roever, and G. Rozenberg, editors, Stepwise Refinement of Distributed Systems. Springer-Verlag, 1990.

[13] Simon S. Lam and A. Udaya Shankar. Protocol verification via projections. IEEE transactions on software engineering, SE-10(2):137-151, July 1984. 
[14] Paul Loewenstein. Reasoning about state-machines in higher-order logic. In M. Leeser and G. Brown, editors, Hardware Specification, Verification and Synthesis: Mathematical Aspects. Springer Verlag, 1990.

[15] Paul Loewenstein and David L. Dill. Verification of a multiprocessor cache protocol using simulation relations and higher-order logic. In Computer-Aided Verification (Proceedings of the CAV90 Workshop), volume 3 of DIMACS Series in Discrete Mathematics and Theoretical Computer Science. American Mathematical Society, 1991.

[16] Nancy A. Lynch and Mark R. Tuttle. Hierarchical correctness proofs for distributed algorithms. Technical Report TR-387, MIT Laboratory for Computer Science, 1987.

[17] Paliath Narendran and Jonathan Stillman. Formal verification of the sobel image processing chip. In G. Birtwistle and P. A. Subrahmanyam, editors, Current Trends in Hardware Verification and Automated Theorem Proving, pages 92-107. SpringerVerlag, 1989.

[18] Tobias Nipkow. Formal verification of data type refinement. In J.W. de Bakker, W.P. De Roever, and G. Rozenberg, editors, Stepwise Refinement of Distributed Systems. Springer-Verlag, 1990.

[19] A. P. Sistla. A complete proof system for proving correctness of non-deterministic safety specifications. Technical Report TC-0060-8-89-378, GTE Laboratories, 1989. 